\title{
ADAPTACIÓN DE UNA ESCALA DE TÁCTICAS DE AUTOPRESENTACIÓN EN UNA MUESTRA MEXICANA*
}

\author{
AlAN GONZÁlEZ \\ https://orcid.org/0000-0002-2225-9019 \\ Universidad Nacional Autónoma de México. Ciudad de México, México \\ SOFÍA RIVERA \\ https://orcid.org/0000-0003-1170-2430 \\ Universidad Nacional Autónoma de México. Ciudad de México, México \\ AlejandRa Domínguez \\ https://orcid.org/0000-0002-2697-8627 \\ Universidad Nacional Autónoma de México \\ Universidad Iberoamericana. Ciudad de México, México \\ Pedro Velasco \\ https://orcid.org/0000-0001-8599-8663 \\ Universidad Nacional Autónoma de México. Ciudad de México, México \\ Correo electrónico: alanjimarez@gmail.com
}

Recibido: 18 de marzo del 2021 / Aceptado: 12 de abril del 2021

doi: https://doi.org/10.26439/persona2021.n024(1).5310

\begin{abstract}
Resumen. La autopresentación permite a las personas mostrar una imagen deseada en un afán de pertenecer a diferentes grupos y sentirse bien; por ello, es relevante contar con instrumentos que permitan medir dicho constructo apropiadamente. Este estudio tuvo por propósito adaptar la escala de tácticas de autopresentación (ETA) en una muestra de adultos de la Ciudad de México y área metropolitana. Participaron 626 voluntarios de entre 18 y 60 años. Se obtuvo un instrumento conformado por siete factores que explican el $52 \%$ de la varianza, con un alfa de Cronbach de .90 y con evidencias convergentes de validez al estar correlacionada con la escala de manejo de impresiones de Bolino y Turnley y la escala de necesidad de aprobación social. En conclusión, existen evidencias de validez y confiabilidad que sustentan el uso de la ETA. Además, se apoyó la premisa de que algunas tácticas pueden configurarse de manera diferente dependiendo del contexto cultural, mientras que otras se presentan análogamente en múltiples culturas.
\end{abstract}

Palabras clave: impresiones / imagen pública / psicometría / validez / confiabilidad

\footnotetext{
* Agradecemos al Consejo Nacional de Ciencia y Tecnología (CONACyT) por la beca 917041 y el financiamiento de esta investigación.
} 


\section{ADAPTATION OF THE SELF-PRESENTATION TACTICS SCALE IN A MEXICAN SAMPLE}

Abstract. Self-presentation allows people to display the desired image to belong to different groups and feel good; for this reason, it is important to count with proper tools for its measurement. The present study aimed to adapt the Self-Presentation Tactics Scale (SPTS) in adults from Mexico City and the metropolitan area with 626 volunteers from 18 to 60 years old. Results showed a 7-factor scale (52\% of the explained variance), with a Cronbach's Alpha of .90 and with convergent evidence of validity as it was correlated with the Bolino and Turnley Impression Management Scale and the Social Approval Need Scale. In conclusion, there are validity and reliability evidence in support of the use of the STPS. Additionally, our psychometric findings support the premise that some tactics can be configured differently depending on the cultural context, while others are presented similarly in multiple cultures.

Keywords: impressions / public image / psychometry / validity / reliability 


\section{INTRODUCCIÓN}

En la literatura se parte de la premisa de que los humanos son seres sociales y tienen la necesidad de ser aceptados e incluidos, así como de buscar y mantener relaciones positivas (Molden, Lucas, Gardner, Dean y Knowles, 2009). Se ha observado que los resultados en la vida (Gross, Debus, Liu, Wang y Kleinmann, 2020), al igual que el éxito y desarrollo de las relaciones, dependen, en parte, de las impresiones que las personas tienen unas de las otras, pues estas moldean la forma en que se evalúan y tratan entre sí. Por ello, las personas se interesan en cómo las ven los demás y con base en ello actúan; es decir, intentan gestionar la imagen que los demás tienen de ellas (Leary y Kowalski, 1990). Esto ha sido denominado autopresentación o manejo de impresiones (Leary y Jongman, 2017).

Desde los primeros estudios, se propuso y demostró que las personas realizan una gran variedad de conductas o tácticas de autopresentación (e. g. Jones y Pittman, 1982; Sandal, Van de Vijver, Bye, Sam, Amponsah y Cakar, 2014) para mostrar diferentes impresiones, dependiendo de su objetivo, el contexto y la audiencia (Leary, Allen y Terry, 2011). Por ejemplo, si la persona quiere agradar puede congraciarse y pedir disculpas. Si el individuo busca parecer competente y ser reconocido, puede autopromocionarse y mostrarse como un ejemplo a seguir. Incluso, bajo ciertas circunstancias y objetivos, una persona puede querer mostrar una imagen negativa como ser peligroso o necesitado, recurriendo a tácticas como la intimidación o súplica.

Se han propuesto múltiples taxonomías para catalogar las variadas formas en que las personas se autopresentan (e. g. Jones y Pittman, 1982; Paulhus y Trapnell, 2008; Smith, Watkins, Burke, Christian, Smith, Hall y Simms, 2013). La clasificación de Tedeschi y Melburg (1984) es una de las más aceptadas en la literatura y divide las tácticas en dos categorías que responden a diferentes propósitos interpersonales. Las tácticas asertivas (e. g. congraciarse e intimidar) hacen referencia a conductas que se llevan a cabo de manera proactiva para mostrar a los demás una imagen particular y deseada. En cambio, las tácticas defensivas (e. g. dar excusas y disculparse) se refieren a las conductas destinadas a defender o restablecer una impresión que fue dañada o que se ve amenazada por un evento o situación.

La autopresentación se ha medido con instrumentos de autoinforme desde múltiples enfoques: unos abordan estilos amplios para manejar las impresiones (e. g. Positive SelfPresentation Scale de Kim y Lee, 2011); otros alguna táctica en particular (e. g. Academic Self-Handicapping Scale de Urdan y Midgley, 2001); mientras que otros se centran en contextos específicos (e. g. Cultural Impression Management Scale-Applicant Form de Sandal et al., 2014). La escala de tácticas de autopresentación (ETA; Lee, Quegley, Nesler, Corbett y Tedeschi, 1999) es un instrumento con evidencias de validez y confiabilidad que resalta sobre otros, ya que toma en cuenta que las personas realizan diferentes 
tácticas para autopresentarse con distintas motivaciones y consecuencias. Por ende, permite estudiar el constructo de forma amplia y evitar la sobregeneralización de los hallazgos. Específicamente, la ETA mide la frecuencia autoinformada de doce tácticas que se clasifican de acuerdo con la propuesta de Tedeschi y Melburg (1984): las tácticas defensivas se componen de las excusas, justificación, deslinde, autosabotaje y apología; en tanto que las tácticas asertivas integran el congraciamiento, súplica, intimidación, designación, mejora, crítica y ejemplificación.

A partir de que la ETA fue creada, se ha usado en múltiples estudios que proporcionaron evidencias de consistencia interna (e. g. Hart, Tortoriello, Richardson y Breeden, 2019; Sadler, Hunger y Miller, 2010). En resumen, la mayoría de las tácticas han obtenido resultados satisfactorios, con excepción del autosabotaje y súplica, las cuales han mostrado coeficientes más bajos que el resto y, en algunos casos, por debajo de lo aceptado en la literatura (e. g. Hogan, 2015).

A pesar de que la ETA ha sido ampliamente utilizada, solo se tiene conocimiento de dos estudios que la adaptaron en otros contextos culturales. En una muestra de Arabia Saudita, Safhi y Teleb (2016) encontraron a través de un análisis factorial exploratorio (AFE) que la ETA se conformó por doce factores que explican el $54 \%$ de la varianza, con un coeficiente alfa de Cronbach de .90 . No obstante, no se especificó si los factores obtenidos en el AFE corresponden a las tácticas originales; además, los resultados de un análisis factorial confirmatorio (AFC) mostraron que los datos obtenidos no presentaron un adecuado ajuste al modelo de doce tácticas distribuidas en asertivas y defensivas $\left(X^{2} / \mathrm{gl}=5.55, \mathrm{RMR}=.13, \mathrm{GFI}=.89, \mathrm{CFI}=.90\right)$.

Serap, Durna y Araz (2019), al adaptar el instrumento en una muestra de adultos turcos, encontraron que las puntuaciones obtenidas en la ETA alcanzaron un coeficiente alfa de Cronbach de .93 y se agruparon en nueve factores que explican el $58 \%$ de la varianza. Las tácticas de congraciamiento, apología, ejemplificación, intimidación y autosabotaje mantuvieron su configuración; mientras que cuatro factores se conformaron por reactivos de dos tácticas: justificación-excusa, deslinde-justificación, mejora-designación y crítica-designación. Serap et al. concluyeron que las diferencias entre las tácticas obtenidas en su estudio y las obtenidas en una muestra estadounidense (Lee et al., 1999) se pueden deber a discrepancias culturales, lo que significaría que las conductas asertivas y defensivas pueden percibirse de manera distinta entre turcos y estadounidenses. Estos resultados y conclusiones son congruentes con los de otros estudios en los que se mostró que algunas tácticas, como la intimidación y la mejora, se pueden usar de manera indistinta en diferentes culturas (Domínguez, Velasco, Aguilera y Contreras, 2019; Sandal et al., 2014); mientras que otras son propias del contexto cultural, y la manera en que se presenten depende de sus valores, normas y expectativas (Bye, Sandal, Van de Vijver, Sam, Cakar y Franke, 2011; Kim, Lee y Gim, 2011). 
Particularmente, México es un país con una cultura de tintes colectivistas (Hofstede, Hofstede y Minkov, 2010), por lo que las relaciones interpersonales juegan un papel destacado en la vida diaria y guardan una estrecha relación con el bienestar y autoconcepto. Por ello, se ha sugerido que la autopresentación es relevante en este contexto cultural, pues permite a las personas mostrar una imagen deseada, en un afán de pertenecer, ser aceptados y sentirse bien (Velasco, Rivera, García y Díaz-Loving, 2018). Esta idea ha sido apoyada por estudios en los que se observó que la autopresentación está vinculada con la autoestima, satisfacción con la vida (González, Velasco y Tavizón, 2017), afecto (Velasco et al., 2018) y la necesidad de aprobación social (Domínguez et al., 2019).

Dado el papel de la autopresentación, es relevante contar con un instrumento que permita obtener una medida válida y confiable en el contexto mexicano de la frecuencia con que los individuos se autopresentan. Sin embargo, aun cuando la ETA fue utilizada previamente en México (González et al., 2017; Velasco y Bonilla, 2020; Velasco et al., 2018), no se han proporcionado evidencias de validez y confiabilidad para su uso para este contexto cultural. Considerando esto, así como las diferencias culturales observadas (e. g. Serap et al. 2019), se resalta la necesidad de explorar el funcionamiento de la ETA y si las tácticas que mide son relevantes para ser estudiadas en México. En tal sentido, los propósitos de este estudio fueron adaptar la ETA en adultos de la Ciudad de México y área metropolitana, y obtener evidencias de validez basadas en la estructura interna y evidencias convergentes de validez, así como estimar empíricamente la confiabilidad de las puntuaciones.

\section{MÉTODO}

\section{Participantes}

Se trabajó con muestreo no probabilístico accidental y para el tamaño de la muestra se consideró tener al menos 10 personas por cada reactivo (Osborn y Costello, 2004). Participaron 630 voluntarios de entre 18 y 60 años $(M=30,52, D E=12,70)$. De la muestra total, el $46,19 \%$ se identificó como hombre, el 52,38 \% como mujer y el 1,43 \% prefirió no especificarlo. En cuanto a su escolaridad, el 46,66 \% tenía estudios universitarios, el $29,52 \%$ de preparatoria, el $14,92 \%$ de secundaria, el $6,34 \%$ de posgrado y el $1,9 \%$ de primaria. Respecto a su ocupación, el 46,50 \% informó trabajar, el 38,57 \% estudiar, el $8,25 \%$ ser ama(o) de casa, el 2,22 \% estar desempleado, el 1,58 \% no estudiaba ni trabajaba y el 0,8\% estaba jubilado.

\section{Instrumentos}

Escala de tácticas de autopresentación (ETA; Lee et al., 1999). Consta de 63 reactivos con un formato de respuesta de nueve puntos (1. Muy infrecuente, 9. Muy frecuente), los cuales 
se distribuyen en 12 tácticas que se agrupan a su vez en asertivas ( $\alpha=.91$; congraciamiento, intimidación, súplica, designación, mejora, crítica y ejemplificación) y defensivas ( $\alpha=$.86; excusas, justificación, deslinde, autosabotaje y apología). El coeficiente alfa de Cronbach para el puntaje total de la ETA es de .93. Se cuenta con evidencias convergentes de validez al estar relacionada positivamente con la escala de automonitoreo. También cuenta con una correlación test-retest de .87 y .88 para ambas dimensiones. La traducción de la ETA se realizó siguiendo las directrices de la Comisión Internacional de los Test para la adaptación de los test de una cultura a otra (International Test Commission, 2017).

Adicionalmente, se aplicaron dos instrumentos para obtener evidencias convergentes de validez:

Escala de Manejo de Impresiones de Bolino y Turnley (EMIBT, 1999). Traducida al español y adaptada en una muestra mexicana por Domínguez et al. (2019). Consta de 22 reactivos con un formato de respuesta tipo Likert de cinco puntos (1. Totalmente en desacuerdo, 5. Totalmente de acuerdo), que se distribuyen en cinco estrategias: congraciamiento $(\alpha=.79)$, súplica $(\alpha=.84)$, autopromoción $(\alpha=.77)$, ejemplificación $(\alpha=.70)$ e intimidación ( $\alpha=$.77). La estructura obtenida por Bolino y Turnley (1999) se confirmó en el estudio de Domínguez et al. a través de un AFC (GFI $=.92, \mathrm{TLI}=.91, \mathrm{CFI}=.92, \mathrm{RMSEA}=.05)$.

Escala de Necesidad de Aprobación Social (ENAS; Domínguez y Van de Vijver, 2014). Consta de 14 reactivos distribuidos en dos dimensiones, una positiva (.74) y una negativa (.71). Esta última se recodifica e interpreta como la aceptación de errores comunes. La escala cuenta con índices de ajuste adecuados a la solución bidimensional (RMSEA $=.05$; $\mathrm{GFI}=.96 ; \mathrm{AGFI}=.95 ; \mathrm{TLI}=.90)$ y con evidencias convergentes de validez al estar relacionada positivamente con los factores de la Escala de Deseabilidad Social de Marlowe-Crowne (1960) y del Inventario de Personalidad NEO-FFI (Costa y McCrae, 1999).

\section{Procedimiento}

La aplicación de los instrumentos se realizó en diferentes lugares públicos del centro, sur, norte y oriente de la Ciudad de México y área metropolitana, como plazas, mercados y parques. El aplicador invitó a las personas a participar en una investigación realizada por parte de la Facultad de Psicología de la Universidad Nacional Autónoma de México con relación a cómo se comportan. Siguiendo los lineamientos del Código Ético del Psicólogo (Sociedad Mexicana de Psicología, 2010), a quienes accedieron a participar se les presentó un consentimiento informado en el que se garantizó que los datos proporcionados serían confidenciales, anónimos y solo utilizados con fines de divulgación científica. Después, el aplicador entregó el formato a los participantes, solicitándoles que respondieran con base en las siguientes instrucciones: 
A continuación, se presentan varias afirmaciones relacionadas con la forma en que actúa. Por favor, señale con qué frecuencia realiza cada uno de los comportamientos, seleccionando el número que mejor lo represente.

El aplicador permaneció cerca de los participantes por cualquier duda que pudieran tener, dándoles el tiempo necesario para contestarlo, que en promedio fue de 30 minutos. Al finalizar, a cada uno se le agradeció su participación.

\section{RESULTADOS}

El análisis de los datos se realizó con el programa estadístico SPSS (versión 23). Para el proceso de validación de la ETA se siguieron los pasos propuestos por Howard (2016), Nunnally y Berstein, (1995) y Reyes-Lagunes y García (2008). Inicialmente, se cumplió con lo propuesto por Reyes-Lagunes y García para determinar qué reactivos serían integrados en el AFE al cumplir con al menos tres de los siguientes criterios: 1) tener frecuencia en todas sus opciones de respuesta, 2) tener una distribución típica, 3) discriminar entre quienes tienen puntuaciones más altas y bajas, y 4) correlacionar lo suficientemente fuerte $(r>.30)$ con el resto de la escala y mantener su consistencia. Para ello, se realizó un análisis de frecuencias con el fin de verificar que todas las opciones de respuesta fueron elegidas y se calculó el coeficiente de asimetría de cada reactivo para conocer la distribución de las respuestas. Se realizó un análisis de discriminación basada en grupos extremos a través de la prueba $t$ de Student con el fin de conocer si los reactivos permitían diferenciar entre personas con puntuaciones más bajas y altas. Se calculó la correlación reactivo-total y el alfa de Cronbach para examinar si la eliminación de algún reactivo ayudaba a aumentar la consistencia interna del instrumento. Dado que todos los reactivos cumplieron con al menos tres de los criterios mencionados, no se eliminó ninguno.

La medida de adecuación muestral de Kaiser-Meyer-Olkin (KMO) y el análisis de esfericidad de Barttlet mostraron que la matriz de correlaciones en la que se basó el AFE fue factorizable $\left(K M O=.89\right.$ y $\left.X^{2}[406]=6973.38, p<.001\right)$. Inicialmente, se realizó un primer AFE por factorización de ejes principales forzando la solución a 12 factores para probar si las tácticas de la versión original de la ETA se presentan en la muestra obtenida. Los resultados mostraron que 11 reactivos no cargaron de manera clara en algún factor (cargas factoriales $<.40$ ), cuatro reactivos tuvieron cargas cruzadas y la táctica crítica únicamente se conformó por un reactivo. Por lo tanto, los factores obtenidos no corresponden a la versión original de la ETA.

Después, se realizó un AFE sin forzarlo a una cantidad de factores determinada. Un análisis de correlación entre los reactivos mostró que la mayoría de las relaciones fueron moderadas (.30 $\geq r \leq .70)$, por lo que se utilizó una rotación oblicua (oblimin directo) con un delta igual a 0 . Los criterios considerados para conservar los reactivos fue que 
tuvieran comunalidades $\geq .30$ y cargas factoriales $\geq .40$ dentro del factor principal $\mathrm{y}<.30$ en el resto, esto para evitar cargas cruzadas. Para la retención de los factores se tomó en cuenta el gráfico de sedimentación, el criterio de Kaiser (autovalores $>1$ ) y que cada uno se conformara por al menos tres reactivos. Adicionalmente, se calculó el coeficiente alfa de Cronbach, tanto para la escala total como por factor, para obtener evidencias acerca de la confiabilidad de las puntuaciones de la ETA. Como se muestra en la tabla 1 , se obtuvo una escala con 29 reactivos distribuidos en siete factores que explicaron el $52,74 \%$ de la varianza total y con un $\alpha=.90$.

Tabla 1

Estructura factorial de la ETA

\begin{tabular}{|c|c|c|c|c|c|c|c|}
\hline \multirow{2}{*}{ Reactivos } & \multicolumn{7}{|c|}{ Factores } \\
\hline & 1 & 2 & 3 & 4 & 5 & 6 & 7 \\
\hline $\begin{array}{l}\text { 9. Expreso las mismas actitudes que los demás } \\
\text { para que me acepten. }\end{array}$ & .58 & & & & & & \\
\hline $\begin{array}{l}\text { 27. Les hago favores a otras personas para poder } \\
\text { agradarles. }\end{array}$ & .57 & & & & & & \\
\hline $\begin{array}{l}\text { 53. Les digo a los demás que son más fuertes o } \\
\text { competentes que yo para que hagan cosas por mí. }\end{array}$ & .57 & & & & & & \\
\hline 11. Uso halagos para ganar favores de los demás. & .47 & & & & & & \\
\hline 62. Halago a los demás para ponerlos de mi lado. & .45 & & & & & & \\
\hline 13. Me disculpo cuando he hecho algo mal. & & .73 & & & & & \\
\hline $\begin{array}{l}\text { 3. Si daño a alguien, me disculpo y prometo no } \\
\text { volver a hacerlo. }\end{array}$ & & .68 & & & & & \\
\hline $\begin{array}{l}\text { 17. Trato de compensar cualquier daño que haya } \\
\text { hecho a otros. }\end{array}$ & & .59 & & & & & \\
\hline 8. Les pido ayuda a los demás. & & .50 & & & & & \\
\hline 23. Trato de ser un ejemplo a seguir. & & & -.90 & & & & \\
\hline $\begin{array}{l}\text { 20. Trato de ser un buen ejemplo para que otros } \\
\text { me imiten. }\end{array}$ & & & -.82 & & & & \\
\hline $\begin{array}{l}\text { 15. Trato de servir como ejemplo sobre cómo una } \\
\text { persona debe comportarse. }\end{array}$ & & & -.74 & & & & \\
\hline $\begin{array}{l}\text { 25. Trato de que otras personas actúen de la } \\
\text { misma manera positiva que yo. }\end{array}$ & & & -.49 & & & & \\
\hline $\begin{array}{l}\text { 58. Hago cosas para que la gente me tenga miedo } \\
\text { y así lograr que hagan lo que yo quiero. }\end{array}$ & & & & .77 & & & \\
\hline 50. Intimido a los demás. & & & & .68 & & & \\
\hline $\begin{array}{l}\text { 1. Me comporto de forma tal que provoco miedo } \\
\text { en las personas. }\end{array}$ & & & & .64 & & & \\
\hline $\begin{array}{l}\text { 2. Uso mi tamaño y fuerza para influir en la gente } \\
\text { cuando lo necesito. }\end{array}$ & & & & .57 & & & \\
\hline
\end{tabular}


(continuación)

31. Amenazo a otras personas cuando creo que eso me ayudará a obtener lo que quiero de ellos.

57. La ansiedad interfiere con mi desempeño.

56. Yo mismo pongo obstáculos en el camino de mi éxito.

12. Me enfermo cuando estoy muy presionado por hacer las cosas bien.

37. Les platico a los demás sobre mis cualidades positivas.

22. Les platico a los demás sobre mis logros positivos.

39. Señalo las cosas positivas que hago cuando los demás no se dan cuenta.

59. Cuando tengo éxito en algo, les enfatizo a los demás lo importante que era.

6. Les cuento a los demás cuando hago bien las tareas que a otros se les dificulta.

5. Justifico mi comportamiento para reducir las reacciones negativas de otras personas.

4. Ofrezco explicaciones antes de hacer algo que otras personas puedan pensar que está mal.

24. Justifico de antemano acciones que a los demás podrían desagradarles.
.53
$-.93$

$-.56$

$-.48$

Nota. Los pesos factoriales $<.40$ han sido omitidos.

Elaboración propia

Los factores obtenidos fueron nombrados y definidos a partir del análisis del significado de los reactivos, así como de la teoría. El congraciamiento $(\alpha=.80)$ se refiere a los esfuerzos que tienen por objetivo ganar la simpatía de los demás y obtener favores. La apología $(\alpha=.75)$ consiste en muestras de remordimiento y peticiones de perdón por algo que se ha dicho o hecho a los demás. La ejemplificación $(\alpha=.85)$ hace referencia a los esfuerzos que realiza un individuo para que los demás lo vean como un modelo a seguir e imitar. La intimidación $(\alpha=.80)$ se define como los esfuerzos de una persona por mostrarse como amenazante, provocando con ello temor e influencia en los demás. El autosabotaje ( $\alpha=.68$ ) se trata de comportamientos que muestran al individuo en desventaja a través de la creación de obstáculos. La autopromoción $(\alpha=.81)$ abarca los esfuerzos a través de los cuales un individuo muestra sus características positivas y logros para sobresalir entre otras personas; mientras que el deslinde $(\alpha=.74)$ se refiere a explicaciones con las cuales un individuo busca evitar que los demás se hagan una imagen negativa de él como consecuencia de la conducta prevista. 
Posteriormente, se realizó un AFE de segundo orden para examinar si las tácticas obtenidas se acomodan de acuerdo con la clasificación de Tedeschi y Melburg (1984) y con la propuesta de Lee et al. (1999). Se encontró una configuración de dos factores correlacionados ( $r=.39$ ) que explican el $48,91 \%$ de la varianza (ver tabla 2 ). Las tácticas de congraciamiento, intimidación, autosabotaje y autopromoción se acomodaron en el primer factor; mientras que la apología, deslinde, ejemplificación y autopromoción conformaron el segundo.

Tabla 2

Análisis factorial de segundo orden de la ETA

\begin{tabular}{llc}
\hline \multirow{2}{*}{ Tácticas } & \multicolumn{2}{c}{ Factores } \\
\cline { 2 - 3 } & 1 & 2 \\
\hline Congraciamiento & .77 & \\
Intimidación & .71 & \\
Autosabotaje & .52 & \\
Autopromoción & .47 & .42 \\
Apología & & .81 \\
Deslinde & & .50 \\
Ejemplificación & & .48 \\
\hline
\end{tabular}

Nota. Los pesos factoriales $<.40$ han sido omitidos.

Elaboración propia

Finalmente, se realizaron análisis de correlación entre los factores de la ETA con las dimensiones de la ENAS y de la EMIBT para obtener evidencias convergentes de validez. En resumen, se encontraron correlaciones positivas y estadísticamente significativas entre el congraciamiento, apología, ejemplificación, intimidación, autosabotaje, autopromoción y deslinde con las dimensiones de la ENAS y de la EMIBT (ver tabla 3). Las correlaciones más altas fueron entre el congraciamiento, intimidación y autopromoción con las estrategias equivalentes medidas por la EMIBT. 
Tabla 3

Correlación entre los factores de la ETA con las dimensiones de la ENAS y de la EMIBT

\begin{tabular}{lccccccccc}
\hline & NAP & NAN & CONB & SUPB & AUTB & EJEB & INTB & M & DE \\
\hline Congraciamiento & .03 & $.48^{* *}$ & $.64^{* *}$ & $.50^{* *}$ & $.36^{* *}$ & $.47^{* *}$ & $.37^{* *}$ & 2,76 & 1,62 \\
Apología & $.25^{* *}$ & -.06 & $.15^{* *}$ & $-.08^{*}$ & $.19^{* *}$ & .00 & $-.08^{*}$ & 5,94 & 1,87 \\
Ejemplificación & $.19^{* *}$ & .06 & $.28^{* *}$ & $.10^{* *}$ & $.42^{* *}$ & $.19^{* *}$ & $.17^{* *}$ & 4,62 & 2,28 \\
Intimidación & $-.13^{* *}$ & $.38^{* *}$ & $.26^{* *}$ & $.40^{* *}$ & $.18^{* *}$ & $.38^{* *}$ & $.61^{* *}$ & 2,32 & 1,52 \\
Autosabotaje & -.06 & $.22^{* *}$ & $.27^{* *}$ & $.23^{* *}$ & $.16^{* *}$ & $.26^{* *}$ & $.27^{* *}$ & 3.63 & 2,04 \\
Autopromoción & .07 & $.23^{* *}$ & $.44^{* *}$ & $.27^{* *}$ & $.61^{* *}$ & $.35^{* *}$ & $.32^{* *}$ & 4,02 & 1,88 \\
Deslinde & $.12^{* *}$ & $.13^{* *}$ & $.33^{* *}$ & $.15^{* *}$ & $.19^{* *}$ & $.21^{* *}$ & $.09^{*}$ & 4,36 & 2,12 \\
\hline$M$ & 3,03 & 2,01 & 2,35 & 1,82 & 2,94 & 1,98 & 1,99 & & \\
$D E$ & 0,83 & 0,75 & 1,00 & 0,82 & 0,95 & 0,78 & 0,86 & & \\
\hline
\end{tabular}

Nota. NAP = dimensión positiva de la ENAS, NAN = dimensión negativa de la ENAS, CONB = congraciamiento de la EMIBT, SUPB = súplica de la EMIBT, AUTB = autopromoción de la EMIBT, EJEB = ejemplificación de la EMIBT, INTB = intimidación de la EMIBT. ${ }^{* *} p \leq .01,{ }^{*} p \leq .05$

Elaboración propia

\section{DISCUSIÓN}

Los propósitos de este estudio fueron adaptar la ETA en adultos y obtener evidencias de validez basadas en la estructura interna y evidencias convergentes de validez, así como estimar empíricamente la confiabilidad de sus puntuaciones. Se observó que la ETA quedó conformada por tácticas consistentes con la literatura y, desde una perspectiva cultural, corresponden a características de la cultura mexicana propuestas por el modelo de Hofstede et al. (2010); sin embargo, no se mantuvieron todas las tácticas de la versión original del instrumento.

Específicamente, la ETA quedó conformada por las tácticas congraciamiento, apología, ejemplificación, intimidación y autosabotaje; además, la táctica denominada deslinde se conglomeró por reactivos de justificación y deslinde, mientras que la autopromoción se conformó por reactivos de mejora y designación. De esta forma, pareciera que hay tácticas como la intimidación y congraciamiento que se presentan sin grandes alteraciones en Estados Unidos (Lee et al., 1999), Turquía (Serap et al., 2019) y México. En cambio, hay otras como el deslinde y la autopromoción que se estructuran de manera particular e, incluso, hay algunas como la súplica y la crítica que no se presentan en algunos contextos culturales como el mexicano. Esto apoya la premisa de que hay ciertas tácticas que, pese a las diferencias culturales, son empleadas en diversos contextos (Sandal et al., 2014); en tanto que otras son propias del contexto cultural y se configuran de manera diferente para adaptarse a sus valores, normas y expectativas (Bye et al., 2011; Kim et al., 2011). 
De acuerdo con el modelo de Hofstede et al. (2010), la cultura mexicana se caracteriza por ser colectivista, jerárquica, masculina y con una alta evitación de la incertidumbre. Esto quiere decir que en esta cultura: 1) se le da un valor preponderante a mostrar una imagen favorable a los demás, establecer y mantener relaciones positivas, evitar conflictos y pertenecer a grupos deseados; 2 ) se acepta que haya un orden jerárquico en el que todos tienen un lugar y se espera que unos tengan mayor poder que otros; 3) se valora la competencia, los logros, el éxito y el reconocimiento; y 4) se realizan comportamientos que reduzcan la incertidumbre, dado que lo desconocido y las situaciones ambiguas provocan ansiedad y malestar. En este sentido, en los mexicanos, el congraciamiento y apología pueden realizarse para mantener la armonía social y agradar a los demás. La intimidación puede efectuarse en un afán de mantener o aumentar la influencia y estatus social. La autopromoción y ejemplificación pueden llevarse a cabo para mantener una impresión favorable, conservar el estatus y obtener reconocimiento. En tanto que el autosabotaje y deslinde pueden usarse en un afán de aumentar la certeza sobre el futuro, en este caso sobre la imagen pública. Autosabotearse ayuda a la persona a esperar que un posible fracaso se asocie a factores externos y el éxito a sus características personales; a la vez que el deslinde le ayuda a tener cierta seguridad de que sus comportamientos no afectarán negativamente la impresión que los demás tienen de ella.

El AFE de segundo orden mostró que la dimensión denominada "Tácticas asertivas" se conformó por el congraciamiento, intimidación, autosabotaje y autopromoción; mientras que la dimensión "Tácticas defensivas" integró la autopromoción, apología, deslinde y ejemplificación. Aun cuando las tácticas obtenidas no se agruparon de acuerdo con lo establecido por Lee et al. (1999), a primera vista encajan con la clasificación de Tedeschi y Melburg (1984), así como con propuestas recientes. Se ha sugerido que el autosabotaje puede usarse como un intento para establecer impresiones, sirviendo como una táctica asertiva (Hart et al., 2019). Asimismo, se ha planteado que la autopromoción puede realizarse de forma tanto proactiva como defensiva (Sandal et al., 2014) e, incluso, se encontró que la ejemplificación también se usa en situaciones en las que la imagen fue dañada o está en peligro, esto como una forma de proteger o recuperar la impresión deseada (Bonner et al., 2017). Sin embargo, en una futura investigación se someterá a prueba la estructura obtenida en este estudio a través de un AFC y se comparará con otros modelos propuestos en la literatura (e. g. Bolino y Turnley, 1999; Lee et al., 1999; Paulhus y Trapnell, 2008; Smith et al., 2013) para comprobar cuál se ajusta mejor a los datos en el contexto mexicano.

Las correlaciones positivas entre las tácticas de la ETA con las dimensiones de la ENAS y la EMIBT sugieren evidencias convergentes de validez. Domínguez et al. (2019) mencionaron que quienes tienen una alta necesidad de aprobación, también tienen una mayor tendencia a manejar sus impresiones dentro de los contextos y demandas sociales para mantener y crear relaciones sociales armoniosas y adaptarse de manera favorable. 
Adicionalmente, el hecho de que las correlaciones entre el congraciamiento, intimidación y autopromoción con sus equivalentes de la EMIBT sean las más altas brinda apoyo de su convergencia. La ejemplificación fue la única táctica que tuvo una baja correlación con su equivalente; no obstante, esto se puede deber a las diferencias en cómo es abordada por cada instrumento: la ETA la define como la tendencia a mostrarse como un ejemplo a seguir (Lee et al., 1999), mientras que la EMIBT la define como autosacrificarse e ir más allá del deber (Bolino y Turnley, 1999).

Por último, este estudio permitió conocer las tácticas que pueden ser relevantes para medir en la Ciudad de México y área metropolitana, pero también brindó información acerca de los reactivos que conforman cada una y sobre cómo se podrían clasificar. No obstante, dadas las diferencias encontradas previamente entre hombres y mujeres en la realización de tácticas de autopresentación (e. g. Sadler et al., 2010), en un siguiente estudio también se realizará un análisis de invarianza para verificar si estos grupos interpretan de la misma forma cada una de las tácticas. Además, debido a la baja consistencia interna de la táctica de autosabotaje, se sugiere que en futuros estudios se revisen los reactivos que conforman esta dimensión y se considere la inclusión de nuevos reactivos que permitan mejorar su confiabilidad y contenido.

En conclusión, este es el primer estudio en proporcionar evidencias de la validez y confiabilidad de la ETA en México (González et al., 2017; Velasco y Bonilla, 2020; Velasco et al., 2018). Contar con este instrumento brindará la posibilidad de conocer la red nomológica de las tácticas de autopresentación en este contexto cultural; además de explorar qué variables promueven la ejecución de las distintas tácticas, así como las consecuencias particulares que puede tener realizar cada táctica para el bienestar y salud mental de personas adultas. Este conocimiento podría ser el punto de partida para el desarrollo de programas y estrategias que promuevan la realización de tácticas que beneficien el bienestar de las personas, a la vez que reduzcan el uso de tácticas con consecuencias negativas.

\section{REFERENCIAS}

Bolino, M., y Turnley, W. (1999). Measuring Impression Management in Organizations: A Scale Development Based on the Jones and Pittman Taxonomy. Organizational Research Methods, 2(2), 187-206. https://doi.org/10.1177/109442819922005

Bonner, J., Greenbaum, R., y Quade, M. (2017). Employee Unethical Behavior to Shame as an Indicator of Self-Image Threat and Exemplification as a Form of Self-Image Protection: The Exacerbating Role of Supervisor Bottom-Line Mentality. Journal of Applied Psychology, 102(8), 1203-1221. https://doi.org/10.1037/apl0000222 
Bye, H., Sandal, G., Van de Vijver, F., Sam, D., Cakar, N., y Franke, G. (2011). Personal Values and Intended Self-Presentation During Job Interviews: A Cross-Cultural Comparison. Applied Psychology: An International Review, 60, 160-182. https://doi. org/10.1111/j.1464-0597.2010.00432.x

Costa, P., y McCrae, R. (1999). NEO PI-R, inventario de personalidad neo revisado. NEO-FFI, inventario neo reducido de cinco factores. TEA Ediciones.

Crowne, D., y Marlowe, D. (1964). The Approval Motive. Wiley.

Domínguez, A., y Van de Vijver, F. J. (2014). An Indigenous Social Desirability Scale. Measurement and Evaluation in Counseling and Development, 47(3), 199-214. https:// doi.org/10.1037/t45076-000

Domínguez, A., Velasco, P., Aguilera, S., y Contreras, M. (2019). Validation of the Bolino and Turnley's Impression Management Scale in a Mexican Sample. Acta de Investigación Psicológica, 9(3), 90-99. https://doi.org/10.22201/fpsi.20074719e. 2019.3.325

González, A., Velasco, P., y Tavizón, B. (2017). Estrategias de manejo de impresiones y su relación con variables psicológicas. Revista Especializada en Ciencias de la Salud, 20(2), 12-21. https://www.medigraphic.com/cgi-bin/new/resumen. cgi?IDARTICULO $=82568$

Gross, C., Debus, M., Liu, Y., Wang, M., y Kleinmann, M. (2020). I am Nice and Capable! How and When Newcomers' Self-Presentation to Their Supervisors Affects Socialization Outcomes. Journal of Applied Psychology. Advance online publication. https://doi.org/10.1037/apl0000817

Hart, W., Tortoriello, G., Richardson, K., y Breeden, C. (2019). Profiles and Profile Comparisons Between Dark Triad Constructs on Self-Presentation Tactic Usage and Tactic Beliefs. Journal of Personality, 87(3), 501-517. https://doi.org/10.1111/ jopy.12411

Hofstede, G., Hofstede, G. J., y Minkov, M. (2010). Cultures and Organizations: Software of the mind (3. ${ }^{a}$ ed.). Mc Graw Hill.

Hogan, T. (2015). Psychological Testing: A Practical Introduction (3. ${ }^{\text {a }}$ ed.). John Wiley \& Sons, Inc.

Howard, M. (2016). A Review of Exploratory Factor Analysis Decisions and Overview of Current Practices: What We Are Doing and How Can We Improve? International Journal of Human-Computer Interaction, 32(1), 51-62. https://doi.org/10.1080/104 47318.2015.1087664

International Test Commission. (2017). ITC Guidelines for Translating and Adapting Tests

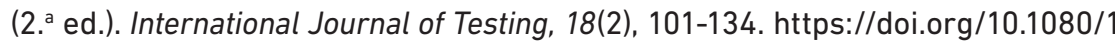
5305058.2017 .139816 
Jones, E., y Pittman, T. (1982). Toward a General Theory of Strategic Self-Presentation. En J. Suls (Ed.), Psychology Perspectives on the Self (pp. 231-262). Erlbaum.

Kim, J., y Lee, J. (2011). The Facebook Paths to Happiness: Effects of the Number of Facebook Friends and Self-Presentation on Subjective Well-Being. Cyberpsychology, Behavior, and Social Networking, 14(6), 359-364. https://doi. org/10.1089/cyber.2010.0374

Kim, J., Lee, S., y Gim, W. (2011). Culture and Self-Presentation: Influence of Social Interactions in an Expected Social Relationship. Asian Journal of Social Psychology, 14, 63-74. https://doi.org/10.1111/j.1467-839X.2010.01332.x

Leary, M., Allen, A., y Terry, M. (2011). Managing Social Images in Naturalistic Versus Laboratory Settings: Implications for Understanding and Studying SelfPresentation. European Journal of Social Psychology, 41(4), 411-421. https://doi. org/10.1002/ejsp.81

Leary, M., y Jongman, K. (2017). Self-Presentation: Signaling Personal and Social Characteristics. En J. Burgoon, N. Magnenat, M. Pantic, y A. Vinciarelli (Eds.), Social Signal Processing (pp. 69-77). Cambridge University Press.

Leary, M., y Kowalski, R. (1990). Impression Management: A Literature Review and Two Component Model. Psychological Bulletin, 107, 34-47. https://doi. org/10.1037/0033-2909.107.1.34

Lee, S., Quigley, B., Nesler, M., Corbett, A., y Tedeschi, J. (1999). Development of a SelfPresentation Tactics Scale. Personality and Individual Differences, 26(4), 701-722. https://doi.org/10.1016/S0191-8869(98)00178-0

Molden, D., Lucas, G., Gardner, W., Dean, K., y Knowles, M. (2009). Motivations for Prevention or Promotion Following Social Exclusion: Being Rejected Versus Being Ignored. Journal of Personality and Social Psychology, 96(2), 415-431. https://doi.org/10.1037/a0012958

Nunnally, J., y Bertstein, I. (1995). Teoría psicométrica. McGraw Hill.

Osborn, J., y Costello, A. (2004). Sample Size and Subject to Item Ratio in Principal Components Analysis. Practical Assessment, Research y Evaluation, 9(11), 1-9. https://doi.org/0.7275/ktzq-jq66

Paulhus, D., y Trapnell, P. (2008). Self-Presentation on Personality Scales: An AgencyCommunion Framework. En O. John, R. Robins, y L. Pervin (Eds.), Handbook of Personality (pp. 492-517). Press.

Reyes-Lagunes, I., y García, L. (2008). Procedimiento de validación psicométrica culturalmente relevante: un ejemplo. En S. Rivera, R. Díaz, R. Sánchez e I. ReyesLagunes (Eds.), La psicología social en México (vol. XII, pp. 625-636). AMEPSO. 
Sadler, M., Hunger, J., y Miller, C. (2010). Personality and Impression Management: Mapping the Multidimensional Personality Questionnaire into 12 Self-Presentation Tactics. Personality and Individual Differences, 48(5), 623-628. https://doi.org/10.1016/j. paid.2009.12.020

Safhi, M., y Teleb, A. (2016). Comparison of Self-Presentation Tactics Between Visually Impaired and Sighted Students. Journal of Arabic Studies in Education y Psychology, 69, 297-314. https://doi.org/10.12816/0022660

Sandal, G., Van de Vijver, F., Bye, H., Sam, D., Amponsah, B., y Cakar, N. (2014). Inteded Self-Presentation Tactics in Job Interviews: A 10-Country Study. Journal of CrossCultural Psychology, 45(6), 1-20. https://doi.org/10.1177/0022022114532353

Serap, O., Durna, G., y Araz, A. (Julio, 2019). The Turkish Adaptation of Self-Presentation Tactics Scale: Preliminary Results [Sesión de póster]. XVI European Congress of Psychology, Moscú, Rusia.

Smith, A., Watkins, M., Burke, M., Christian, M., Smith, C., Hall, A., y Simms, S. (2013). Gendered Influence A Gender Role Perspective on the Use and Effectiveness of Influence Tactics. Journal of Management, 39(5), 1156-1183. https://doi. org/10.1177/0149206313478183

Sociedad Mexicana de Psicología. (2010). Código Ético del Psicólogo (5. a ed.). Editorial Trillas.

Tedeschim, J., y Melburg V. (1984). Impression Management and Influence in the Organization. En S. Bacharach y E. Lawler (Eds.), Research in the Sociology of Organizations (pp. 31-58). JAI.

Urdan, T., y Midgley, C. (2001). Academic Self-Handicapping: What we Know, What More There is to Learn. Educational Psychology Review, 13, 115-138. https://doi. org/10.1023/A:1009061303214

Velasco, P. y Bonilla, C. (2020). Efectos de la discriminación percibida en una muestra de jóvenes mexicanos. En I. Blanca (Ed.), Aportaciones de la psicología al bienestar del ser humano (pp. 121-135). Universidad Nacional Autónoma de México.

Velasco, P., Rivera, S., García, M., y Díaz-Loving, R. (2018). Discriminación, manejo de impresiones y habilidades para la vida como predictores del bienestar. En R. DíazLoving, L. Reyes-Lagunes y F. López. La psicología social en México (vol. XVII). AMEPSO. 